\title{
3 Research Square

\section{Prevalence and Antimicrobial Susceptibility Pattern of Neisseria Gonorrhoeae from Selected Public Health Centers, Addis Ababa, Ethiopia.}

\section{Enaniye Ayalew}

Addis Ababa University

Mistire Wolde

Addis Ababa University

\section{Semira Ebrahim}

Ethiopian Public Health Institute

\section{Elias Seyoum}

Ethiopian Public Health Institute

\section{Zerihun Woldesenbet}

Yekatit 12 Hospital and Medical College

Surafel Fentaw Dinku ( $\nabla$ sura4f@gmail.com )

Ethiopian Public Health Institute

\section{Research Article}

Keywords: Sexually transmitted infection, Neisseria gonorrhoeae, antibiotic susceptibility pattern, Ethiopia.

Posted Date: February 17th, 2022

DOI: https://doi.org/10.21203/rs.3.rs-1244871/v1

License: (9) This work is licensed under a Creative Commons Attribution 4.0 International License. Read Full License 


\section{Abstract}

Background: Neisseria gonorrhea (NG) is gram negative diplococcic bacteria that cause most important sexually transmitted infections (STIs) worldwide. In most developing countries including Ethiopia, NG infections are diagnosed syndromically, and its antibiotic susceptibility is rarely tested. Thus, the objective of the present study was to determine the prevalence and antimicrobial susceptibility patterns of $N$. gonorrhoeae among syndrome diagnosed and treated cases attending selected public health centers, in Addis Ababa, Ethiopia.

Methods: a cross-sectional study was conducted from April 2019 to March 2020, at selected health centers STIs clinics in Addis Ababa, Ethiopia. Urethral discharge or vaginal discharge specimens were cultured on Modified Thayer Martín media and suspected gonococcal colonies were confirmed using Oxidase, Superoxol tests. Antimicrobial susceptibility testing was performed by Minimum Inhibitory Concentration (MIC) using concentration gradient strips (E-test) of the same antimicrobial agents of ciprofloxacin, penicillin, Ceftriaxone, Azytromicin, Cefoxitin, and Spectinomicin

Results: Among the total 325 NG suspects 163 (50\%) were culture positive. Among the culture positive cases 126 were male and 37 females. Around $4 \%$ of isolates are non-susceptible to Ceftriaxone which is the current antimicrobial agent used for urethral discharge/ vaginal discharge syndrome treatment.

Conclusion: In the current study, the proportion of $N$. gonorrhoeae culture positivity rate among male was found to be high. The antimicrobial agent ceftriaxone used for the treatment of gonococccal isolates found to become non susceptible. Antimicrobial agents outside of ciprofloxacin and natural penicillin antimicrobial agents are eligible for the national STI syndromic management.

\section{Introduction}

Neisseria gonorrhea (NG) is gram- negative intracellular diplococci, non-capsulated \& Oxidase positive organisms. $N$. gonorrhoeae is one of the most common sexually transmitted diseases in developing countries (1). According to World Health Organization (WHO) estimate in 2016, around 87 million new gonorrhea infections recorded worldwide (2). The bacteria can reside at different anatomical sites such as the urethra, endocervix, pharynx, or rectum and transmitted from infected host to the other during unsafe sexual intercourse $(3,4)$.

Syndromic management of sexually transmitted infections are becoming increasingly challenging due to antimicrobial resistance (AMR) to various classes of available antibiotic therapy (5). In the syndromic management of NG, ceftriaxone is the last remaining option for empirical first-line antimicrobial monotherapy, in most part of the world. (6). However, NG developing resistance to ceftriaxone at different regions worldwide with the proportion of varying extensively, from $1.3 \%$ to $55.8 \%$ (7).

Therefore regular assessment and updates on prevalence of NG, as well as antimicrobial sensitivity pattern of the bacteria so important, mainly for those developing courtiers including Ethiopia, which 
practiced only sydromic diagnosis and treatment of the diseases. Besides, in order to control and prevent occurrence of NG prevalence and antimicrobial worldwide, such kind of studies are so essential.

\section{Materials And Methods}

\section{Study Area}

This research was carried out in three health centers; Janmeda health center woreda 06, Arada health center woreda 10, and Gerji health center woreda 13, Addis Ababa, Ethiopia. The selected health centers are administered under the Addis Ababa Health Bureau in Addis Ababa, Ethiopia.

\section{Study Design and Period}

A cross-sectional study was conducted to compare the Syndromic approach versus etiologic based approach on STI at a selected Health center in Addis Ababa, Ethiopia, from April 2019-March 2020.

\section{Source Population}

All clients who visited in selected health center outpatient department, OPD for medical service with a compliant of sign and symptoms of urethral /vaginal discharge.

\section{Study Population}

The study populations were patients in the selected health facilities of Addis Ababa who were participated in the study. All Out Patient Department (OPD) clients for STI according to WHO standard Syndromic approach STI management guideline between April 2019-March 2020 were included in this study.

\section{Inclusion Criteria}

Clients who visited the study site OPD and have a history of urethral /vaginal discharge Neisseria gonorrhoeae and willing to participate in the study.

\section{Exclusion Criteria}

Clients who have no signs and symptoms of Neisseria gonorrhoeae and patients having a history of taking antibiotics for the past 10 days are to be excluded.

\section{Sample Size Calculation and Sampling Method}

In this study sample size was calculated by using single population proportion formula with a confidence interval of $95 \%$ and $5 \%$ marginal error by taking prevalence of the case $(74.1 \%)$ from a study conducted at Gondar town hospital and health centers, Ethiopia (8). And by adding $10 \%$ of calculated sample sizes for contingency, finally, the sample size was determined to be three hundred twenty-five. And convenience sampling method is used $(9,10)$. 


\section{Sample collection}

Appropriate ureteral and vaginal discharge and blood (serum) samples was collected by a trained clinical nurse. The ureteral and vaginal discharges were transported by Amies with charcoal transport media.

\section{Laboratory Analysis}

A sterile cotton-tipped swab was used to obtain swab specimens. Then sterile Dacron swabs tipped applicator were used to collect urethral secretions. The swabs were inoculated on in-house prepared Modified Thayer Martin Agar plates made of Gonococcal agar base supplemented with isovitalex (vitox); vancomycin, colistin, nystatin, and trimethoprim (VCNT); and synthetic hemoglobin (Oxoid and BBL).The inoculated plates were incubated on the site using candle jar and transported to the Ethiopian Public Health Institute (EPHI), Clinical Bacteriology and Mycology Reference Laboratory within the same day of collection. Swab was rolled onto a microscopy slide, labeled, heat fixed, placed in a slide box and sent to EPHI for Gram-stain analysis. Gram stain was performed to look for polymorph nuclear leucocytes with Gram-negative intracellular diplococci microscopically. Then the isolates were identified as Neisseria gonorrhoeae by presumptive diagnosis method based on colony morphology, Gram staining, catalase test, Oxidase test, and superoxide test.

\section{Culture and identification:}

In the clinical bacteriology laboratory, inoculated plates were incubated at $35^{\circ} \mathrm{C}$ in carbon dioxide enriched environment (5-8 \% C02) for 72 hours inspecting every day for the growth of small, translucent and non-pigmented colonies. Gram negative diplococcic convex, glistening, elevated, mucoid colony characteristics and oxidase, catalase and supercool $(30 \% \mathrm{H} 2 \mathrm{O} 2)$ positive were considered as probable $N$. gonorrhoeae. Antimicrobial susceptibility testing was performed by on enriched GC agar (Oxoid Ltd) plus 1\% BBL Isovitalex Enrichment. Minimum Inhibitory Concentration (MIC) was done using concentration gradient strips (E-test) of the same antibiotics. The range of inhibition zones and MIC for each type of antibiotic disk were interpreted according to Clinical Laboratory Standard Institute (CLSI) guideline (11). N. gonorrhoeae reference strain ATCC 49226 was used a positive control.

\section{Data Analysis and Interpretation}

For data entry and analysis, SPSS (the Statistical Package for Social Sciences for Windows) version 23 statistical software was used. Bivariate analysis was used to determine Socio-demographic Characteristics versus Neisseria gonorrhoeae, percentage was calculated.

\section{Ethical Considerations}

This study was ethically cleared by Research and Ethics Committee of the Addis Ababa University College of Health Sciences Department of Medical Laboratory Technology and Addis Ababa Public Health Research and Emergency Management Directorate. 
At the enrollment visit, all participants with urethral discharge or vaginal discharge were given written consent diagnosed according to the syndromic treatment guidelines currently approved in Ethiopia. Those who are eligible ( $>18$ years of age) and willing to participate in the study were asked using structured questionnaire for their demographic and behavioral data. All data were kept confidential anonymously. Participants were informed of their rights to resign from the study at any level of the study without any restriction if they were feeling uncomfortable in the study. Their results of all positive individuals were reported to the respective health facilities for better management of the patients.

\section{Results}

\section{Socio-demographic characteristics}

A total of 325 STI symptomatically diagnosed and treated patients were enrolled in the study, and of them 167 (51.4\%) were males. The majority of the study participants were between the ages of 26 and 35. (54.5\%).According to the marital status analysis, the majority of people were single $178(54.8 \%)$; whereas only $19(5.8 \%)$ were illiterate. Out of 325 participants, one hundred seventy eight (54.8\%) were singles in their marital status. Table1.

Of all the specimens analyzed, only $163(50 \%)$ gonococcal isolates were identified. Among these gonococcal isolates 126 were male and 37 females. Table 2.

The present study has revealed that $N$. gonorrhoeae isolates recovered from this study have shown high level of resistance to natural penicillin and fluoroquinolone class of antibiotics but best susceptible for cephalosporin, macrolides, and spectinomycin in Ethiopia. Figure1.

\section{Discussion}

The present study showed that among sydromic diagnosed cases, more than half of participants had laboratory- confirmed gonococcal infection. A similar community based Syndromic versus Laboratory Diagnosis of Sexually Transmitted Infections study conducted in Northern Tanzania, indicated that of the total participated 1,520 women, gonorrhea was confirmed in $0.2 \%$ cases(12). Another study conducted in India indicated that out of 1120 Syndromic STI cases only 409 (36.5\%) cases were confirmed in the laboratory for pathogens (13). Another study conducted in Jimma (western Ethiopia) private clinics indicated that of the total 1315 samples examined, 31(9.8\%) were confirmed to have gonococcal infection (14). The findings from the present study compared with others has relatively high culture positivity rate of gonococcal etiologies. The difference might be due to, the study setting, study participates (more male participants), laboratory diagnostic approaches, and disease prevalence of the study areas. In this study the culture positivity rate compared with other studies it has high prevalent. The difference might be nature of participants in this study. In this study more participants were male and the positivity rate from urethral discharge higher than vaginal discharge (7). 
The present study has revealed that $N$. gonorrhoeae isolates recovered from this study have shown high level of resistance to commonly prescribed fluoroquinolone class of antibiotics in Ethiopia. This finding is in agreement with other study conducted previously in Ethiopia which reported a resistance level of $67 \%$ (15). The Non-susceptibility to ceftriaxone which is used for the national N. gonorrhoeae treatment was detected in $5 \%$ all of the isolates tested during the study period. This finding is in agreement with other studies conducted in different part of Ethiopia $(16,17$, and 18). This indicated that the resistance is increasing through time compared with the susceptibility pattern of this antimicrobial agent six years before (. Antimicrobial agents tested in this study except ciprofloxacin and natural penicillin have susceptibility pattern of greater than $95 \%$ which could be used for the treatment of syndromic management.

\section{Conclusion}

In the current study, the proportion of $\mathrm{N}$. gonorrhoeae culture positivity rate among male was found to be high. The antimicrobial agent ceftriaxone used for the treatment of gonococccal isolates found to become non susceptible. Still the antimicrobial agent is competent agent for the syndromic management of urethral / vaginal discharge syndrome. Antimicrobial agents outside of ciprofloxacin and natural penicillin antimicrobial agents are eligible for the national STI syndromic management. The data found in this study could be input for the national syndromic management.

\section{Abbreviations}

CDC....... Center for Disease Control

NG......... Neisseria gonorrhoeae

OPD....... Out Patient Department

STDs....... Sexually transmitted diseases

STI........ Sexually transmitted infection

STIs....... Sexually transmitted infections

TP.......... Treponema palladium

VDRL..... Venereal Disease Research Laboratory

\section{Declarations}

\section{Conflict of interest}

The authors declare that there is no competing interest. 
All methods were performed in accordance with the relevant guidelines and regulations

Written informed consent obtained from human participants

\section{Funding source}

Addis Ababa University College of Health Sciences Department of Medical Laboratory Technology and Ethiopian Public Health Institute Anti-Microbial Resistance Surveillance Program provided minimal financial support.

\section{Authors' contribution}

- Enaniye Ayalew is the first researcher who prepared the proposal, analyzed and interprets the finding, and prepared the manusc

- Zerihun Woldesenbet reviewed the proposal, assisted in data collection and review of the manusc

- Semira Ebrahim and Elias Seyoum assisted the identification and interpretation processes, and review the manuscript

- Mistire Wolde- reviewers of the proposal, main research work, and reviewed the final version of the manuscript

- Surafel Fentaw reviewed the proposal, assisted in data collection and prepared the manuscript; and correspondence author

- All authors have read and reviewed the final version of the manusc

\section{Acknowledgment}

The authors would like to thank all the study participants and staff at the data collection site health centers. Besides, authors duly acknowledge EPHI microbiology laboratory staff, Gondar University microbiology laboratory staff, and Addis Ababa University School of health since college. Finally, the authors thank Addis Ababa University and Ethiopian Public Health Institute Anti-Microbial Resistance Surveillance Program for partial financial support of the study.

\section{Operational definitions}

Non-susceptible $N$. gonorrhoeae isolates were defined as those that are not sensitive to the antibiotic tested for susceptibility, i.e., those isolates exhibiting resistance or intermediate resistance.

\section{Ethic's approval and consent to participate}

The study was approved by the Research and Ethics Committee of the Addis Ababa University College of Health Sciences Department of Medical Laboratory Science (Reference No. SMLT. 200/11/19 and Addis Ababa Public Health Research and Emergency Management Directorate. The privacy and confidentiality of the study participants' data were protected (Reference No. AAM/36433/227. 


\section{Consent for publication}

This research article did not have individual person's data in any form, or the case of children. Thus separate consent's form did not attach. But, if needed we can provide. However, we have got ethical clearance for the mentioned in the declarations. Participants are agreed the data to be published.

\section{Availability of supporting data}

All necessary data related with the research presented within the article can be provided up on request if needed.

\section{References}

1. M.Lesmana,C.I.Lebron,D.Taslimetal.,"Invitroantibiotic susceptibility of Neisseria gonorrhoeae in Jakarta, Indonesia," Antimicrobial Agents and Chemotherapy, vol. 45, no. 1, pp. 359-362, 2001.

2. World Health Organization, Report on global sexually transmitted infection surveillance 2018 , Word Heal Organ, 2018.

3. Koumans EH, Johnson RE, Knapp JS, St. Louis ME. Laboratory testing for Neisseria gonorrhoeae by recently introduced nonculture tests: a performance review with clinical and public health considerations. Clinical infectious diseases. 1998; 27(5):1171-80.

4. Harvard Health Topics A-Z, Gonorrhea. Medically reviewed on Jun 15, 2018.

5. M. W. Kivata, M. Mbuchi, F. Eyase et al. "Plasmid mediated penicillin and tetracycline resistance among Neisseria gonorrhoeae isolates from Kenya," BMC Infectious Diseases, vol. 20, pp. 1-11, 2020.

6. M. Unemo and W. M. Shafer, "Antimicrobial resistance in Neisseria gonorrhoeae in the 21st century: past, evolution, and future," Clinical Microbiology Reviews, vol. 27, no. 3, pp. 587-613, 2014.

7. M. M. Lahra, "Surveillance of antibiotic resistance in Neisseria gonorrhoeae in the WHO Western Pacific and South East Asian Regions, 2010," Communicable Diseases Intelligence Quarterly Report, vol. 36, no. 1, pp. 95-100, 2012.

8. Geremew RA, Agizie BM, Bashaw AA, Seid ME, Yeshanew AG. Prevalence of selected sexually transmitted infection and associated factors among symptomatic patients attending Gondar Town hospitals and health Centers. Ethiopian journal of health sciences. 2017; 27(6):589-600.

9. 9. Workowski KA, Bolan GA. sexually transmitted diseases treatment guidelines, 2015. MMWR. Recommendations and reports: Morbidity and mortality weekly report. Recommendations and 2015; $6: 1$

10. Etikan I, Musa SA, Alkassim RS. Comparison of convenience sampling and purposive sampling. American Journal of Theoretical and Applied Statistics. 2016 5(1):1-4.

11. CLSI (2020). Performance standards for antimicrobial susceptibility testing. $30^{\text {th }}$ edition. Clinical and Laboratory Standard Institute 
12. Cheng Y,. Paintsil E., Ghebremichael M. Syndromic versus Laboratory Diagnosis of Sexually Transmitted Infections in Men in Moshi District of Tanzania. Hindawi, AIDS Research and Treatment. 2020.

13. Tankhiwale SS, ChavanSP. Comparative study of Syndromic and etiological diagnosis of sexually transmitted infection except human immunodeficiency virus in sexually transmitted infection and reproductive tract infection clinic attendees in central India. International Journal of Medicine and PublicHealth.2013; 3(4).

14. Sahile A., Teshager L.,Fekadie M. ,Gashaw M. Prevalence and Antimicrobial Susceptibility Patterns of Neisseria gonorrhoeae among Suspected Patients Attending Private Clinics in Jimma, Ethiopia. Hindawi International Journal of Microbiology Volume 2020, Article ID 7672024, 6 pages https://doi.org/10.1155/2020/7672024.

15. Surafel F., Rajiha A.,Negga A., Meseret A., Yonas B. and Eyasu T. Antimicrobial susceptibility profile of Gonococcal isolates obtained from men presenting with urethral discharge in Addis Ababa, Ethiopia: Implications for national syndromic treatment guideline. https://doi.org/10.1371/journal.pone.0233753

16. Tibebu M, Shibabaw A, Medhin G and Kassu A (2013). Neisseria gonorrhoeae non-susceptible to cephalosporins and quinolones in Northwest Ethiopia. BMC Infectious Diseases 13:415.

17. Ali S, Sewunet T, Sahlemariam Z, Kibru G. Neisseria gonorrhoeae among suspects of sexually transmitted infection in Gambella hospital, Ethiopia: risk factors and drug resistance. BMC Res Notes. 2016 Sep 13;9(1):439

18. Yeshanew A.G., Geremew R.A. Neisseria gonorrhoae and their antimicrobial susceptibility patterns among symptomatic patients from Gondar town, north West Ethiopia. Antimicrobial Resistance and Infection Control (2018) 7:85 https://doi.org/10.1186/s13756-018-0376-3

19. M. Unemo and W. M. Shafer, "Antimicrobial resistance in Neisseria gonorrhoeae in the 21st century: past, evolution, and future," Clinical Microbiology Reviews, vol. 27, no. 3, pp. 587-613, 2014.

20. A.P.R.daCosta-Lourenço, K.T.B.dosSantos, B.M.Moreira, S. E. L. Fracalanzza, and R. R. Bonelli, "Antimicrobial resistance in Neisseria gonorrhoeae: history, molecular mechanisms and epidemiological aspects of an emerging global threat," Brazilian Journal of Microbiology, vol. 48, no. 4, pp. 617-628, 2017.

21. Oree K., Naicker M. , Maise H.C., Tinarwo P. , Abba S. Antimicrobial Susceptibility Patterns in Neisseria gonorrhoeae Isolated from South African Pregnant Women. Hindawi Infectious Diseases in Obstetrics and Gynecology Volume 2021, Article ID 6684680, 6 pages https://doi.org/10.1155/2021/6684680

\section{Tables}

Table1: Frequency and Percent of Socio-demographic characteristics of study participants at selected health centers in Addis Ababa Ethiopia from April 2019- March 2020. 


\begin{tabular}{llll} 
Variable & & Frequency & Percent \\
\hline Gender & Male & 167 & 51.4 \\
\cline { 2 - 4 } & Female & 158 & 48.6 \\
\hline Age & $18-25$ & 101 & 31.1 \\
\cline { 2 - 4 } & $26-35$ & 177 & 54.5 \\
\cline { 2 - 4 } Educational status & $>35$ & 47 & 14.5 \\
\cline { 2 - 4 } & Illiterate & 19 & 5.8 \\
\hline \multirow{2}{*}{ Marital status } & Literate & 306 & 94.2 \\
& Single & 178 & 54.8 \\
\cline { 2 - 4 } & Married & 135 & 41.5 \\
\cline { 2 - 4 } & Divorced & 11 & 3.4 \\
\cline { 2 - 4 } & Widowed & 1 & 0.3
\end{tabular}

Table 2: Culture positivity rate of study participants at selected health centers in Addis Ababa Ethiopia from April 2019- March 2020.

\begin{tabular}{lcc} 
Specimen $\mathbf{n = 3 2 5}$ & Culture positivity $\mathbf{n = 1 6 2}$ & Percent \\
\hline Urethral discharge (167) & 126 & $75(126 / 167)$ \\
\hline Vaginal discharge (158) & 37 & $23(37 / 158)$ \\
\hline Total & 162 & $50(162 / 325)$
\end{tabular}

\section{Figures}




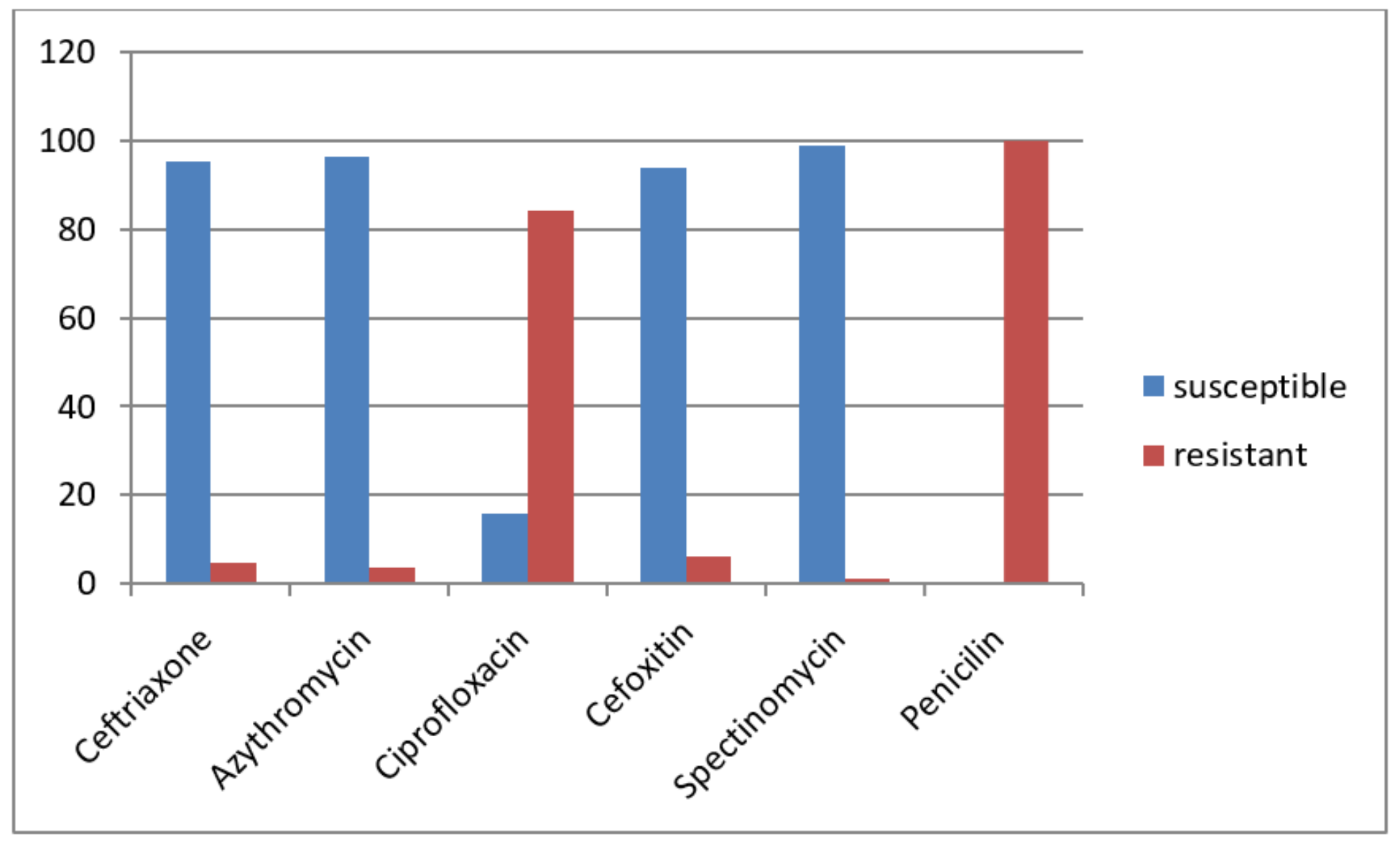

Figure 1

Antimicrobial susceptibility pattern of Neisseria gonorrhoeae participants at selected health centers in Addis Ababa Ethiopia from April 2019- March 2020.

\section{Supplementary Files}

This is a list of supplementary files associated with this preprint. Click to download.

- ASTFORN.gonorrhoae2.docx 